\title{
Bènaco o Benaco?
}

\section{Enzo Caffarelli}

PUBBLICATO: 11 FEBBRAIO 2020

\section{Quesito:}

Una lettrice ci scrive a proposito dell'accento da porsi su Benaco, altro nome del lago di Garda: dice che sente spesso pronunciarlo con l'accento sulla seconda vocale, mentre a sua detta i veronesi abitanti del lago mettono l'accento sulla prima vocale.

\section{Bénaco o Benaco?}

P otrebbe apparire logico accogliere la dizione più diffusa del limnonimo [ovvero il nome di uno specchio d'acqua dolce] e di alcuni toponimi circostanti, Benáco parossitono e censurare garbatamente la ritrazione dell'accento, attribuendola ora a ignoranza, ora a un fenomeno linguistico in parte dovuto all'influenza del tedesco moderno che ha per esempio condizionato la pronuncia errata di numerosi cognomi veneti (Fúrlan, Bénetton, Pádoan, Trévisan ecc. che sono invece tronchi). La forma proparossitona Bénaco si ascolta molto spesso in bocca ai veronesi e talora anche ai trentini e ai lombardi orientali.

Nel sito della Comunità del Garda si legge il parere del prof. Giorgio Bargioni che ben riassume l'opinione di tanti veronesi, quando scrive:
Almeno nei territori della sponda veronese è molto frequente sentir pronunciare, anche da persone impegnate culturalmente e durante manifestazioni di vario genere, il nome del nostro lago Bénaco con l'accento sulla e, anziché Benáco, come sarebbe corretto [...]. Midomando se la comunità non potesse trovare il modo di suggerire sommessamente ai propri aderenti di cercare di correggere quell'accento, anche tenendo conto del fatto che i non gardesani, e in particolare gli stranieri, conoscono la pronuncia corretta.

E nel sito si commenta:

Purtroppo, non rimane che consolarci con la giusta pronuncia dei turisti, anche perché bresciani,
mantovani e trentini non sono più dotti dei veronesi, il male è comune, mentre sulle iniziative che la
comunità potrà prendere per contrastare l'uso errato della parola, apriamo volentieri un dibattito.

In breve, Benaco deriva dal celtico *bennacus 'cornuto', per la forma del lago o per i promontori che si inoltrano nelle sue acque, passato al latino come benacus, mentre Garda è il longobardo warda, 'posto di guarda, punto di osservazione', in origine località abitata, oggi comune di Garda, nome poi esteso al lago. Le due forme sono rimaste in competizione per secoli e del resto non sorprende: il fenomeno è comune ad altri laghi, il Maggiore o Verbano, di Como o Lario, d'Iseo o Sebino, di Bolsena o Volsinio ecc.

Ma le cose non sono così semplici. Giovan Battista Pellegrini ha ricostruito le possibili etimologie del limnonimo nella sua Toponomastica italiana (Milano, Hoepli I990, p. II9); l'origine sarebbe celtica, ma le prime due righe del paragrafo dedicate dal grande toponomasta riportano Benācus lacus nome classico accanto alle attestazioni proparossitone Bé̈nakos in Polibio e in Strabone: infatti in attico la 
sequenza prosodica (breve-lunga-breve) sposta obbligatoriamente l'accento dalla penultima alla terzultima sillaba, mentre al contrario il mantovano Virgilio, nelle Georgiche e nell'Eneide, o Catullo di Sirmione, nelle sue poesie, consideravano tonica la penultima. Né si può ignorare l'autorevolezza di Dante che, nel canto XX dell'Inferno, cita due volte il lago con evidente accentazione parossitona: "Suso in Italia bella giace un laco | a piè e l'Alpe che serra Lamagna | sopra Tiralli, ch'a nome Benaco" (vv. 6I-63) e "Ivi convien che tutto quanto caschi $\mid$ ciò che 'n grembo a Benaco star non può | e fassi fiume giù per verdi paschi" ( vv. 73-75). Al Benaco sono dedicati anche poemetti cinquecenteschi in latino di Pietro Bembo e di Giorgio Jodoco Bergano; il limnonimo è citato inoltre in unode barbara del Carducci e nel componimento dannunziano Per la coppia del Benaco. Agli aviatori navali.

Come mi suggerisce il glottologo Guido Borghi (Università degli Studi di Genova), alla base del nome è per la precisione il gallico *Bènnákŏs, che se intedescato suonerebbe *Bennach (cfr. Brembach/Premesa, località di San Michele di Castelrotto in Alto Adige), mentre se si fosse conservato nella tradizione orale romanza sarebbe *Benágo o *Banágo (cfr. Brusago in Val di Piné in Trentino). Ciò detto, la conclusione è che Benaco è una forma soltanto letteraria, recuperata pienamente in epoca umanistica (a parte la Commedia) e la sua accentazione riflette appunto le norme prosodiche latine o greche a seconda della preferenza di chi lo nomina; mentre, in chi non si pone la questione, prevale il prestigio della fonte da cui lo apprende.

In sintesi, la pronuncia parossitona largamente più diffusa in Italia appare la più convincente, anche per le autorevoli fonti latine e di quelle italiane dei secoli scorsi, che da quelle dipendevano. Tuttavia un a diversa dizione, proparossitona, non può far gridare allo scandalo. Semmai occorrerebbe approfondire le motivazioni per le quali proprio intorno al Lago di Garda e ai comuni che nella denominazione ne contengono il nome latino - San Felice del Benaco (Brescia) e Torri del Benaco (Verona), sulle opposte sponde dell'area gardesana - si registra frequentemente la pronuncia minoritaria.

Come appendice andrà detto che, al pari di altri toponimi antichi, latineggianti o grecizzanti, suppletivi, anche Benaco è entrato da tempo nell'ambito dell'odonimia e nella crematonimia [odonimi sono i nomi delle strade e crematonimi i nomi delle attività commerciali]; a parte 33 vie e vicoli Benaco e 6 vie o piazze Benacense (dati SEAT/Pagine Gialle Italia 20r3), possono citarsi un Hotel Benaco a Desenzano del Garda (Brescia), a Garda (Verona) a Nago-Torbole (Trento), la Locanda del Benaco a Salò (Brescia) e qui e altrove anche l'agenzia immobiliare Benaco, l'agenzia di viaggi BenacoVacanze, l'agenzia pratiche automobilistiche Benaco a Peschiera del Garda (Verona) e, fuori zona, il ristorante Stile Benaco e l'Osteria Benaco a Roma (in via Benaco).

\author{
Cita come: \\ Enzo Caffarelli, Bènaco o Benaco?, "Italiano digitale”, 2019, XII, 2020/1 (gennaio-marzo) \\ DOI: $10.35948 / 2532-9006 / 2020.3228$ \\ Copyright 2019 Accademia della Crusca \\ Pubblicato con licenza creative commons CC BY-NC-ND
}

